\title{
STARSHAPEDNESS OF LEVEL SETS FOR THE OBSTACLE PROBLEM AND FOR THE CAPACITORY POTENTIAL PROBLEM ${ }^{1}$
}

\author{
BERNHARD KAWOHL
}

\begin{abstract}
If the geometry of the data in the obstacle problem and the capacitory potential problem is starshaped then so are the solutions. The proofs are based on appropriate maximum principles.
\end{abstract}

Introduction. Consider the one-dimensional obstacle problem: Find a function $u \in H_{0}^{1}([a, b]), u(x) \geqslant \psi(x)$ on $[a, b], u^{\prime \prime}(x)=0$ if $u(x)>\psi(x)$, where $\psi:[a, b] \rightarrow \mathbf{R}$ is a given "obstacle" with the properties $\psi(a)<0, \psi(b)<0$ and $\psi$ " $<0$, i.e. $\psi$ is strictly concave. Obviously the solution $u$ has the following geometrical properties:

(i) $u$ is concave.

(ii) The coincidence set $I:=\{x \in[a, b] \mid u(x)=\psi(x)\}$ is an interval.

(iii) The level sets $\Omega_{c}:=\{x \in[a, b] \mid u(x) \geqslant c\}$ are intervals.

Not all of these properties are inherited to higher dimensions. In fact property (i) already fails for two dimensions. In order to extend (ii) and (iii) to more than one dimension we have to find out which property of an interval is inherited to higher dimensions. Intervals enjoy many properties; they are, for example, convex, starshaped and simply connected.

If we replace the word "interval" by "simply connected domain", then property (ii) extends to two dimensions [7,p. $173 \mathrm{ff}$ ]. In general, however, the coincidence set is neither starshaped nor convex as was pointed out by D. Kinderlehrer [6, p. 14].

In this paper the word "interval" is replaced by "starshaped domain" and then property (iii) remains valid for arbitrary dimensions.

1. The obstacle problem. Let $\Omega \subset \mathbf{R}^{n}$ be a bounded open set with smooth boundary $\partial \Omega$. Let $\psi: \bar{\Omega} \rightarrow \mathbf{R}$ be an "obstacle" satisfying

$$
\begin{aligned}
& \psi \in C^{1,1}(\Omega), \quad \max _{\Omega} \psi=\psi_{\max }>0,\left.\quad \psi\right|_{\partial \Omega}<0 \text { and } \\
& \left(x-x^{0}\right) \nabla \psi(x)<0 \text { for fixed } x^{0} \in \Omega \text { and any } x \in \bar{\Omega} \backslash\left\{x^{0}\right\} .
\end{aligned}
$$

The last property is satisfied if $\psi$ is concave and has a maximum in $x^{0}$, or if the level sets $\Omega_{c}:=\{x \in \Omega \mid \psi(x) \geqslant c\}$ of $\psi$ are starshaped with respect to $x^{0}$.

Received by the editors December 7, 1982 and, in revised form, March 22, 1983

1980 Mathematics Subject Classification. Primary 35J65, 35B50, 35R35.

'These and other results have been presented to the Oberwolfach-Conference on Partial Differential Equations on March 4, 1983. This research was partially supported by the Deutsche Forschungsgemeinschaft (DFG). 
Let $K:=\left\{v \in H_{0}^{1}(\Omega) \mid u \geqslant \psi\right.$ in $\left.\Omega\right\}$, let $f(x, u) \in C^{1, \alpha}\left(\bar{\Omega} \times\left[0, \psi_{\max }\right]\right)$ with

$$
\begin{gathered}
f(x, 0)=0, \quad f(x, u) \geqslant 0, \quad f_{u}(x, u) \geqslant 0, \\
\left(x-x^{0}\right) \nabla_{x} f(x, u) \geqslant 0 \quad \text { on } \bar{\Omega} \times\left[0, \psi_{\max }\right],
\end{gathered}
$$

and let $u \in K$ be the solution of the variational inequality

$$
\int_{\Omega}\{\nabla u(\nabla v-\nabla u)+f(x, u)(v-u)\} d x \geqslant 0 \quad \text { for all } v \in K .
$$

It is well known that a unique solution $u \in C^{1}(\Omega) \cap H^{2, s}(\Omega)$ with $s<\infty$ exists [2, p. $17 ; 6$, p. 108].

THEOREM 1. If $\Omega$ is starshaped with respect to $x^{0}$ and if the functions $\psi$ and $f$ satisfy conditions (1) and (2), then the level sets of the solution $u(x)$ to the variational inequality (3) are starshaped with respect to $x^{0}$. In particular, $u$ has only one critical point $x^{0}$.

Proof. Formally, $u$ satisfies the equations

$$
\begin{aligned}
\Delta u & =f(x, u) \quad \text { in the noncoincidence set } \Omega \backslash I, \text { and } \\
u & =\psi \quad \text { in the coincidence set } I .
\end{aligned}
$$

The maximum principle $\left[5\right.$, p. 206f] implies $0 \leqslant u(x) \leqslant \psi_{\max }$ in $\Omega$. Due to the starshapedness of the domain $\Omega$ the function $\left(x-x^{0}\right) \nabla u(x)$ is nonpositive on $\partial \Omega$. Furthermore, $\left(x-x^{0}\right) \nabla u(x)=\left(x-x^{0}\right) \nabla \psi(x)<0$ on $I \backslash\left\{x^{0}\right\}$ because of (1), so that $\left(x-x^{0}\right) \nabla u(x)<0$ on $\partial(\Omega \backslash I)$. Since $u \in C^{3, \alpha}(\Omega \backslash I)$ we may compute the Laplacian of $\left(x-x^{0}\right) \nabla u(x)$ in $\Omega \backslash I$ and obtain

$$
\text { div } \operatorname{grad}\left\{\left(x-x^{0}\right) \nabla u(x)\right\}=\left(x-x^{0}\right) \nabla(\Delta u(x))+2 \Delta u(x) \quad \text { in } \Omega \backslash I .
$$

Assumption (2) now implies

$$
-\Delta\left\{\left(x-x^{0}\right) \nabla u(x)\right\}+f_{u}(x, u)\left\{\left(x-x^{0}\right) \nabla u(x)\right\} \leqslant 0 \quad \text { in } \Omega \backslash I,
$$

so that by the strong maximum principle [5, p. 34] $\left(x-x^{0}\right) \nabla u(x)<0$ in $\Omega \backslash\left\{x^{0}\right\}$.

REMARK 1. It seems to be an open problem, whether a similar statement holds for convex domains $\Omega$ and concave obstacles $\psi$, namely that the level sets of $u$ are convex.

2. The capacitory potential problem. Let $\Omega_{1}$ and $\Omega_{0}$ be bounded open subsets of $\mathbf{R}^{n}$ with smooth boundaries $\Gamma_{1}$ and $\Gamma_{0}$ respectively. Let $\Omega_{1} \Subset \Omega_{0}$. Denote $\Omega_{0} \backslash \bar{\Omega}_{1}$ by $\Omega$ and suppose that $u \in C^{3}(\Omega)$ is a solution to the capacitory potential problem

$$
\begin{aligned}
\Delta u(x) & =f(x, u) \quad \text { in } \Omega, \\
u(x) & =1 \quad \text { on } \Gamma_{1}, \\
u(x) & =0 \quad \text { on } \Gamma_{0},
\end{aligned}
$$

where $f(x, u) \in C^{1, \alpha}(\bar{\Omega} \times[0,1])$ satisfies

$$
\begin{gathered}
f(x, 0)=0, \quad f(x, u) \geqslant 0, \quad f_{u}(x, u) \geqslant 0, \\
\left(x-x^{0}\right) \nabla_{x} f(x, u) \geqslant 0 \quad \text { on } \bar{\Omega} \times[0,1] .
\end{gathered}
$$

Observe that (2) and (2') differ only in the admissible interval for the variable $u$. 
THEOREM 2. If $\Omega_{1}$ and $\Omega_{0}$ are starshaped with respect to $x^{0} \in \Omega_{1}$, and if $f$ satisfies condition $\left(2^{\prime}\right)$, then the level sets of the solution $u(x)$ to the capacitory potential problem (4) are starshaped with respect to $x^{0}$.

For the proof we use the same arguments as in Theorem 1 and show that $\left(x-x^{0}\right) \nabla u(x)<0$ in $\Omega$.

REMARK 2. The resemblance between Theorems 1 and 2 is not surprising. If $\psi$ is defined as the characteristic function of $\Omega_{1}$ on $\Omega_{0}$, then the solution of the capacitory potential problem can be interpreted as a solution to an obstacle problem on $\Omega_{0}$ with irregular obstacle.

REMARK 3. The question of convex level lines for convex $\Omega_{0}$ and $\Omega_{1}$ has been investigated in $[8$, p. $203 ; 1 ; 3 ; 4$, p. 553]. We want to sketch a new proof for the result of L. Caffarelli and J. Spruck, which does not require the analyticity of $u$ and which uses the structure of $f$ in a more transparent way,

THEOREM 3. If $\Omega_{0}$ and $\Omega_{1}$ are convex and if $u \in C^{2, \alpha}(\Omega)$ is a solution to the capacitory potential problem,

$$
\begin{aligned}
\Delta u(x) & =f(u) \quad \text { in } \Omega, \\
u(x) & =1 \quad \text { on } \Gamma_{1}, \\
u(x) & =0 \quad \text { on } \Gamma_{0},
\end{aligned}
$$

where $f(u) \in C^{1, \alpha}([0,1])$ satisfies

$$
f(0)=0, f(u) \geqslant 0, f_{u}(u)>0 \quad \text { on }\left[\begin{array}{ll}
0 & 1
\end{array}\right],
$$

then the level sets of $u$ are all convex.

The proof makes use of the fact that a continuous function $u: \Omega_{0} \rightarrow \mathbf{R}$ defined on a convex domain $\Omega_{0}$ has convex level sets iff

$$
Q\left(x_{1}, x_{2}\right):=u\left(\left(x_{1}+x_{2}\right) / 2\right)-\min \left\{u\left(x_{1}\right), u\left(x_{2}\right)\right\} \geqslant 0 \quad \text { in } \Omega_{0} \times \Omega_{0} .
$$

Let us extend the solution $u$ of the capacitory potential problem by the constant 1 on $\bar{\Omega}_{1}$, then $u$ is well defined on $\Omega_{0}$, and suppose that the function $Q$ has a global negative minimum in $\left(x_{1}, x_{2}\right) \in \bar{\Omega}_{0} \times \bar{\Omega}_{0}$. Because of Theorem 2 we can exclude that $x_{1}, x_{2}$ or $\left(x_{1}+x_{2}\right) / 2$ is not in $\Omega=\Omega_{0} \backslash \bar{\Omega}_{1}$. We now apply the following lemma.

LEMMA. Let $\Omega \subset \mathbf{R}^{n}$ be a bounded domain, let $u \in C^{2, \alpha}(\Omega)$ and suppose

(i) There exists $x^{0} \in \mathbf{R}^{n}$ such that $\left(x-x^{0}\right) \nabla u(x)<0$ for $x^{0} \neq x \in \Omega$.

(ii) If $Q$ has a global negative minimum in $\left(x_{1}, x_{2}\right) \in \Omega \times \Omega$, then $\left(x_{1}+x_{2}\right) / 2 \in \Omega$.

Then if $Q$ has a global negative minimum in $\Omega \times \Omega$, the minimum is attained in a pair $\left(x_{1}, x_{2}\right) \in \Omega \times \Omega$ for which (a)-(c) hold.

(a) $u\left(x_{1}\right)=u\left(x_{2}\right)$.

(b) The gradients of $u$ in those three points are mutually parallel and point in the same direction, i.e. $\nabla u\left(x_{1}\right) \uparrow \uparrow \nabla u\left(\left(x_{1}+x_{2}\right) / 2\right) \uparrow \uparrow \nabla u\left(x_{2}\right)$, and $a=2 b c /(b+c)=$ $\mu b+(1-\mu) c$, where $\mu=c /(b+c), a=\left|\nabla u\left(\left(x_{1}+x_{2}\right) / 2\right)\right|, b=\left|\nabla u\left(x_{1}\right)\right|$ and $c=\left|\nabla u\left(x_{2}\right)\right|$.

(c) $\left(\mu / b^{2}\right) \Delta u\left(x_{1}\right)+\left((1-\mu) / c^{2}\right) \Delta u\left(x_{2}\right) \leqslant\left(1 / a^{2}\right) \Delta u\left(\left(x_{1}+x_{2}\right) / 2\right)$. 
Let us mention that statements (a) and (b) remain true if the starshapedness assumption (i) is replaced by superharmonicity $(\Delta u<0$ in $\Omega$ ) and that (ii) holds for convex domains $\Omega$.

In order to conclude the proof of Theorem 3 we have to observe that the Lemma and the positivity and monotonicity of $f$ imply

$$
\frac{\mu}{b^{2}}+\frac{1-\mu}{c^{2}}<\frac{1}{(\mu b+(1-\mu) c)^{2}},
$$

a contradiction to the convexity of the function $x^{-2}$.

The proof of the Lemma is based on the simple observation that $Q$ can be rewritten as

$$
Q\left(x_{1}, x_{2}\right)=u\left(\left(x_{1}+x_{2}\right) / 2\right)-\frac{1}{2} u\left(x_{1}\right)-\frac{1}{2} u\left(x_{2}\right)+\frac{1}{2}\left|u\left(x_{1}\right)-u\left(x_{2}\right)\right|,
$$

and approximated by a sufficiently smooth function

$$
Q_{\varepsilon}\left(x_{1}, x_{2}\right)=u\left(\left(x_{1}+x_{2}\right) / 2\right)-\frac{1}{2} u\left(x_{1}\right)-\frac{1}{2} u\left(x_{2}\right)+j_{\varepsilon}\left(u\left(x_{1}\right)-u\left(x_{2}\right)\right),
$$

where $j_{\varepsilon}(s)$ is a regularized approximation to the function $|s| / 2$. If $Q$ has a negative minimum then so does $Q_{\varepsilon}$ in $\left(x_{1}^{\varepsilon}, x_{2}^{\varepsilon}\right)$ and for $\varepsilon \rightarrow 0$ there exists a minimizing (sub)sequence converging to some $\left(x_{1}, x_{2}\right) \in \Omega \times \Omega$.

To prove (a), we assume that $u\left(x_{1}\right)<u\left(x_{2}\right)$. Then $Q$ is $C^{2, \alpha}$ locally near $\left(x_{1}, x_{2}\right)$ and the gradient with respect to $x_{2}$ has to vanish, i.e. $u\left(\left(x_{1}+x_{2}\right) / 2\right)=0$ in contrast to (i).

To prove (b) we first compute the gradients of $Q_{\varepsilon}$ and observe that $\left|j_{\varepsilon}^{\prime}\right| \leqslant 1 / 2$. This leads to the parallelity statement. Elimination of $j_{\varepsilon}^{\prime}$ yields the relation between $a, b$ and $c$.

To prove (c) we have to minimize the function

$$
C\left(x_{1}, x_{2}\right):=u\left(\left(x_{1}+x_{2}\right) / 2\right)-\frac{1}{2} u\left(x_{1}\right)-\frac{1}{2} u\left(x_{2}\right)
$$

under the equality constraint $u\left(x_{1}\right)=u\left(x_{2}\right)$. This can be done in a fashion similar to [1] and implies the desired inequality (c).

\section{REFERENCES}

1. C. Borell, Brownian motion in a convex ring and quasi-concavity, Comm. Math. Phys. 86 (1982). 143-147.

2. H. Brezis, Problemes unilateraux, J. Math. Pures Appl. 51 (1972), 1-172.

3. L. A. Caffarelli and J. Spruck, Convexity properties of solutions to some classical variational problems, Comm. Partial Differential Equations 7 (1982), 1337-1379.

4. A. Friedman, Variational principles and free boundary problems, Wiley, New York, 1982.

5. D. Gilbarg and N. S. Trudinger, Elliptic partial differential equations of second order, Springer-Verlag, Berlin, 1977.

6. D. Kinderlehrer, Variational inequalities and free boundary problems, Bull. Amer. Math. Soc. 84 (1978), 7-26.

7. D. Kinderlehrer and G. Stampacchia, An introduction to variational inequalities and their applications, Academic Press, New York, 1980.

8. G. Polya and G. Szegö, Isoperimetric inequalities in mathematical phisics, Princeton Univ. Press, Princeton, N. J., 1951.

Institut fUr Angewandte Mathematik, Universität Erlangen, Martensstrasse 4, D 8520 ERLANGEN, West Germany

Current address: Lefschetz Center for Dynamical Systems, Brown University, Providence, Rhode Island 02912 